(1)

CrossMark

\title{
Pleural diseases and COVID-19: ubi fumus, ibi ignis
}

\author{
José M. Porcel (10 \\ Affiliation: Pleural Medicine Unit, Dept of Internal Medicine, Arnau de Vilanova University Hospital, IRBLleida, \\ University of Lleida, Lleida, Spain. \\ Correspondence: José M. Porcel, Pleural Medicine Unit, Dept of Internal Medicine, Arnau de Vilanova \\ University Hospital, Avda Alcalde Rovira Roure 80, 25198 Lleida, Spain. E-mail: jporcelplayahoo.es
}

@ERSpublications

There is both direct and circumstantial evidence that SARS-CoV-2 is responsible for the generation of pleural effusions and secondary spontaneous pneumothorax/pneumomediastinum https://bit.ly/ $3 g Z q A 7 Z$

Cite this article as: Porcel JM. Pleural diseases and COVID-19: ubi fumus, ibi ignis. Eur Respir J 2020; 56 : 2003308 [https://doi.org/10.1183/13993003.03308-2020].

More than 45000 articles in the PubMed database and around 3200 studies registered in ClinicalTrials.gov, of which greater than half are clinical trials, are the result of ongoing and relentless research into the global pandemic nature of an acute respiratory disease caused by the severe acute respiratory syndrome coronavirus 2 (SARS-CoV-2), which made its initial appearance in December 2019 in China. As of 28 August 2020, the total confirmed cases of coronavirus disease 2019 (COVID-19) surpasses 24.5 million, with more than 830 000 global deaths [1]. An estimated $40 \%$ to $45 \%$ of persons infected with SARS-CoV-2 will remain asymptomatic, but they can transmit the virus to others for an extended period, perhaps longer than 14 days [2]. The primary presentation of symptomatic infection is that of an influenza-like illness or viral pneumonia, with about $20 \%$ of these patients developing severe or critical manifestations [3].

Pleural disease encompasses pleural effusion and pneumothorax. In the context of COVID-19, both are considered to be so atypical or unusual that they should compel clinicians to seek alternative diagnoses [4]. This might be challenged by new observations, including those of MArTinelu et al. [5] in this issue of the European Respiratory Journal. According to several systematic reviews [6-10], pooled prevalences of pleural thickening and pleural effusions on computed tomography (CT) scans are approximately $38 \%$ and $5 \%$, respectively (table 1). However, the frequency of pleural effusions varies with age and disease severity. For example, in an Iranian study of 552 COVID-19 symptomatic patients, CT detected pleural effusions in $7.6 \%$ of cases overall; a percentage which was significantly higher in those over 50 years of age versus under (10\% versus 5.2\%; $\mathrm{p}=0.037)$ [11]. Additionally, in a multicentre study of 476 patients with COVID-19, more patients in the critical than in the severe or moderate disease groups had pleural effusions on CT images at admission $(18 \%, 7.4 \%$ and $3.1 \%$, respectively; $\mathrm{p}<0.001)$ [12]. It may be argued that most, if not all, of these pleural effusions may be secondary to comorbid conditions rather than being directly related to the viral infection. About half of COVID-19 patients have comorbidities [12, 13], including pre-existing cardiovascular diseases (12.6\%) or heart failure (6.5\%) [14]. Moreover, more than $25 \%$ of infected patients exhibit myocardial injury of potential inflammatory pathogenesis (a cause of impaired cardiac function) as demonstrated by elevation of troponin T levels [14] and, at least $20 \%$ develop heart failure during disease course [15]. On the other hand, the incidence of pulmonary embolism was $8.3 \%$ among 1240 infected patients who were subjected to a CT pulmonary angiography [16]. And

Received: 28 Aug 2020 | Accepted: 30 Aug 2020

Copyright CERS 2020. This version is distributed under the terms of the Creative Commons Attribution NonCommercial Licence 4.0. 
TABLE 1 Prevalence of pleural effusions and thickening on chest computed tomography imaging of adult coronavirus disease 2019 patients: relevant systematic reviews

\begin{tabular}{|c|c|c|c|c|}
\hline Systematic review & Studies $\mathbf{n}$ & Patients $\mathrm{n}$ & Pleural thickening ${ }^{\#}$ & Pleural effusion \\
\hline Adams et al. [6] & 28 & 3466 & $34.7 \%$ & $5.2 \%$ \\
\hline BAO et al. [7] & 13 & 2738 & $52.5 \%$ & $5.8 \%$ \\
\hline OJHA et al. [8] & 45 & 4410 & $41.7 \%$ & $5.0 \%$ \\
\hline Zheng et al. [9] & 15 & 2150 & ND & $3.0 \%$ \\
\hline Zнu et al. [10] & 34 & 4121 & $27.1 \%$ & $5.3 \%$ \\
\hline
\end{tabular}

\#: pleural thickening adjacent to underlying lung involvement. ND: not done.

both, heart failure and pulmonary embolism [17], in addition to respiratory co-infections [18], are well-recognised aetiologies of pleural effusions. Despite the fact that a certain number of patients may have pleural effusions derived from the preceding causes, SARS-CoV-2 undoubtedly gives rise to fluid formation since it has been detected by RT-PCR in pleural fluid specimens [19, 20].

Non-COVID-19 viral pneumonias share many CT features with COVID-19, except for a higher prevalence of pleural effusions. In a systematic review of 33 studies, comprising 1911 patients equally distributed between COVID-19 and non-COVID-19 viral pneumonias (mainly influenza A and adenovirus), pleural effusions were observed in $3 \%$ and $25 \%$ of the cases, respectively [21]. Regarding other beta-coronaviruses, very few small series on CT appearances have been reported and, therefore, firm conclusions cannot be drawn. The true prevalence of pleural effusions in SARS-CoV-1 infections is conflicting, ranging from two (6.8\%) of 29 patients [22] to seven (26\%) of 27 [23] in two different reports. In one study of 55 patients infected with Middle East respiratory syndrome coronavirus (MERS-CoV), pleural effusions were visible in the chest radiographs of 17 (31\%) and represented a poor prognostic indicator [24]. A previous retrospective study by the same authors found that nine (60\%) of 15 patients with MERS-CoV had pleural effusions on CT [25].

MARTinelli et al. [5] describe the occurrence of pneumothorax and pneumomediastinum as important, though infrequent, clinical features of COVID-19 [5]. In their retrospective study, clinical details were reported for 60 cases of pneumothoraces that occurred among an estimated 6574 COVID-19 admissions across multiple centres in the UK. This represents an incidence of $0.91 \%$, which is consistent with previous observations. For instance, in a series of 92 deceased patients with COVID-19, one death was attributed to pneumothorax (1.1\%) [26]. Similarly, this complication was described in one (1\%) of 99 patients by others [27], while seven spontaneous air leaks (five pneumomediastinum and two pneumothoraces) were found as part of 976 COVID-19 cases (0.7\%) in an American institution [28]. Notably, of the 60 pneumothorax patients reported in this issue, $75 \%$ were older than 50 years, and more than half had never smoked, which is difficult to reconcile with the primary spontaneous variety [5]. Also, one-third of the patients were not submitted to invasive or non-invasive mechanical ventilatory support at the time of diagnosis, thus ruling out iatrogenic causes [5]. Consequently, the existence of a true secondary spontaneous pneumothorax due to SARS-CoV-2 should be admitted, as in other respiratory infections. For example, about $1 \%$ of patients with active tuberculosis [29], up to $9 \%$ of those with Pneumocystis jirovecci pneumonia [30], and $1.7 \%$ of SARS-CoV-1 infected subjects [31] may develop pneumothorax. As far as MERS-CoV is concerned, the clinical course of nine (16\%) of 55 patients complicated with pneumothorax, but all of them were intubated and so, the contribution of barotrauma and/or volutrauma was probably meaningful [24]. The pathological basis for pneumothorax in non-ventilated COVID-19 patients is uncertain. While not usually seen in autopsy reports, lung cavitation and/or cystic lesions unrelated to the use of mechanical ventilation can occur in COVID-19 [32]. The severe cough that may be associated with COVID-19 and the corresponding increase in intrapulmonary pressure may precipitate the rupture of viral-induced damaged alveoli of peripheral location and pneumothorax formation. Pneumothorax should be managed following standard guidelines, wearing personal protective equipment for chest tube placement and considering strategies to minimise droplet exposure via the chest drain circuit (e.g. connecting anti-viral filters on suction ports or adding bleach to the water seal chamber) [33].

Many of the preceding statements for pneumothorax in the setting of SARS-CoV-2 infection hold true for pneumomediastinum. It is a rare entity, frequently related to mechanical ventilation, which may also appear spontaneously in the context of respiratory infections, like influenza [34]. In the series reported by Martineli et al. [5], only 1 (9\%) of 11 pneumomediastinum cases was labelled as spontaneous [5]. It appears, however, to be a more frequent characteristic feature of SARS-CoV-1 infection, with two separate 
series of 75 and 112 patients reporting a $12 \%$ incidence of this complication $[35,36]$. Pneumothorax and pneumomediastinum, in addition to pulmonary embolism or progression of the primary disease, should be considered in any COVID-19 patient with acute respiratory deterioration.

In brief, there is direct evidence that SARS-CoV-2 infection produces pleural effusion, and circumstantial evidence that it may cause secondary spontaneous pneumothorax and pneumomediastinum. Although alternative explanations primarily related to comorbidities and iatrogenic effects should always be sought, the Latin aphorism "ubi fumus, ibi ignis" (where there's smoke, there's fire) is perfectly applicable to pleural involvement in the setting of COVID-19.

Conflict of interest: None declared.

\section{References}

1 John Hopkins University and Medicine. COVID-19 Dashboard by the Center for Systems Science and Engineering (CSSE) at Johns Hopkins University ( JHU). https://coronavirus.jhu.edu/map.html

2 Oran DP, Topol EJ. Prevalence of asymptomatic SARS-CoV-2 infection: a narrative review. Ann Intern Med 2020; 173: 362-367.

3 Wu Z, McGoogan JM. Characteristics of and important lessons from the coronavirus disease 2019 (COVID-19) outbreak in China: summary of a report of 72314 cases from the Chinese Center for Disease Control and Prevention. JAMA 2020; 323: 1239-1242.

4 Simpson S, Kay FU, Abbara S, et al. Radiological Society of North America Expert Consensus Statement on reporting chest CT findings related to COVID-19. Endorsed by the Society of Thoracic Radiology, the American College of Radiology, and RSNA - Secondary Publication. J Thorac Imaging 2020; 35: 219-227.

5 Martinelli AW, Ingle T, Newman J, et al. COVID-19 and pneumothorax: a multicentre retrospective case series. Eur Respir J 2020; 56: 2002697.

6 Adams HJA, Kwee TC, Yakar D, et al. Chest CT imaging signature of COVID-19 infection: in pursuit of the scientific evidence. Chest 2020; 158: 1885-1895.

7 Bao C, Liu X, Zhang H, et al. Coronavirus disease 2019 (COVID-19) CT findings: a systematic review and meta-analysis. J Am Coll Radiol 2020; 17: 701-709.

8 Ojha V, Mani A, Pandey NN, et al. CT in coronavirus disease 2019 (COVID-19): a systematic review of chest CT findings in 4410 adult patients. Eur Radiol 2020; 30: 6129-6138.

9 Zheng Y, Wang L, Ben S. Meta-analysis of chest CT features of patients with COVID-19 pneumonia. J Med Virol 2020; in press [https://doi.org/10.1002/jmv.26218].

10 Zhu J, Zhong Z, Li H, et al. CT imaging features of 4121 patients with COVID-19: a meta-analysis. J Med Virol 2020; 92: 891-902.

11 Majidi H, Bani-Mostafavi ES, Mardanshahi Z, et al. High-resolution computed tomography finding in 552 patients with symptomatic COVID-19: first report from north of Iran. Emerg Radiol 2020; in press [https://doi. org/10.1007/s10140-020-01819-9].

12 Feng Y, Ling Y, Bai T, et al. COVID-19 with different severities: a multicenter study of clinical features. Am J Respir Crit Care Med 2020; 201: 1380-1388.

13 Razzaghi $\mathrm{H}$, Wang $\mathrm{Y}, \mathrm{Lu} \mathrm{H}$, et al. Estimated county-level prevalence of selected underlying medical conditions associated with increased risk for severe COVID-19 illness - United States, 2018. MMWR Morb Mortal Wkly Rep 2020; 69: 945-950

14 Momtazmanesh S, Shobeiri P, Hanaei S, et al. Cardiovascular disease in COVID-19: a systematic review and meta-analysis of 10,898 patients and proposal of a triage risk stratification tool. Egypt Heart J 2020; 72: 41.

15 Shafi AMA, Shaikh SA, Shirke MM, et al. Cardiac manifestations in COVID-19 patients-A systematic review. J Card Surg 2020; 35: 1988-2008.

16 Fauvel C, Weizman O, Trimaille A, et al. Pulmonary embolism in COVID-19 patients: a French multicentre cohort study. Eur Heart J 2020; 41: 3058-3068.

17 Porcel JM, Esquerda A, Vives M, et al. Etiology of pleural effusions: analysis of more than 3,000 consecutive thoracenteses. Arch Bronconeumol 2014; 50: 161-165.

18 Lansbury L, Lim B, Baskaran V, et al. Co-infections in people with COVID-19: a systematic review and meta-analysis. J Infect 2020; 81: 266-275.

19 Mei F, Bonifazi M, Menzo S, et al. First detection of SARS-CoV-2 by real-time reverse transcriptase-polymerase chain reaction assay in pleural fluid. Chest 2020; 158: E143-E146.

20 Turriziani O, Sciandra I, Mazzuti L, et al. SARS-CoV-2 diagnostics in the virology laboratory of a University Hospital in Rome during the lockdown period. J Med Virol 2020; in press [https://doi.org/10.1002/jmv.26332].

21 Altmayer S, Zanon M, Pacini GS, et al. Comparison of the computed tomography findings in COVID-19 and other viral pneumonia in immunocompetent adults: a systematic review and meta-analysis. Eur Radiol 2020; 30: 6485-6496.

22 Müller NL, Ooi GC, Khong PL, et al. High-resolution CT findings of severe acute respiratory syndrome at presentation and after admission. AJR Am J Roentgenol 2004; 182: 39-44.

23 Chan MS, Chan IY, Fung KH, et al. High-resolution CT findings in patients with severe acute respiratory syndrome: a pattern-based approach. AJR Am J Roentgenol 2004; 182: 49-56.

24 Das KM, Lee EY, Al Jawder SE, et al. Acute Middle East respiratory syndrome coronavirus: temporal lung changes observed on the chest radiographs of 55 patients. AJR Am J Roentgenol 2015; 205: W267-W274.

25 Das KM, Lee EY, Enani MA, et al. CT correlation with outcomes in 15 patients with acute Middle East respiratory syndrome coronavirus. AJR Am J Roentgenol 2015; 204: 736-742.

26 Yang F, Shi S, Zhu J, et al. Analysis of 92 deceased patients with COVID-19. J Med Virol 2020; in press [https:// doi.org/10.1002/jmv.25891].

27 Chen N, Zhou M, Dong X, et al. Epidemiological and clinical characteristics of 99 cases of 2019 novel coronavirus pneumonia in Wuhan, China: a descriptive study. Lancet 2020; 395: 507-513. 
28 Eperjesiova B, Hart E, Shokr M, et al. Spontaneous pneumomediastinum/pneumothorax in patients With COVID-19. Cureus 2020; 12: e8996.

29 Freixinet JL, Caminero JA, Marchena J, et al. Spontaneous pneumothorax and tuberculosis: Long-term follow-up. Eur Respir J 2011; 38: 126-131.

30 Liu CJ, Lee TF, Ruan SY, et al. Clinical characteristics, treatment outcomes, and prognostic factors of Pneumocystis pneumonia in non-HIV-infected patients. Infect Drug Resist 2019; 12: 1457-1467.

31 Sihoe AD, Wong RH, Lee AT, et al. Severe acute respiratory syndrome complicated by spontaneous pneumothorax. Chest 2004; 125: 2345-2351.

32 Liu K, Zeng Y, Xie P, et al. COVID-19 with cystic features on computed tomography: a case report. Medicine (Baltimore) 2020; 99: e20175.

33 Pieracci FM, Burlew CC, Spain D, et al. Tube thoracostomy during the COVID-19 pandemic: guidance and recommendations from the AAST Acute Care Surgery and Critical Care Committees. Trauma Surg Acute Care Open 2020; 5: e000498.

34 Onigbinde SO, Ojo AS, Fleary L, et al. Chest computed tomography findings in COVID-19 and influenza: a narrative review. Biomed Res Int 2020; 2020: 6928368

35 Peiris JS, Chu CM, Cheng VC, et al. Clinical progression and viral load in a community outbreak of coronavirus-associated SARS pneumonia: a prospective study. Lancet 2003; 361: 1767-1772.

36 Chu CM, Leung YY, Hui JY, et al. Spontaneous pneumomediastinum in patients with severe acute respiratory syndrome. Eur Respir J 2004; 23: 802-804. 